\title{
The role of molecular imaging in the frame of the revised dementia with Lewy body criteria
}

\author{
Stelvio Sestini ${ }^{1,14} \cdot$ Pierpaolo Alongi ${ }^{2,14} \cdot$ Valentina Berti ${ }^{3,14} \cdot$ Maria Lucia Calcagni ${ }^{4,14} \cdot$ Diego Cecchin $^{5,14,15}$. \\ Agostino Chiaravalloti $^{6,14}$. Andrea Chincarini ${ }^{7,14}$. Angelina Cistaro ${ }^{8,14}$. Ugo Paolo Guerra ${ }^{9,14}$. Sabina Pappatà ${ }^{10,14}$. \\ Pietro Tiraboschi ${ }^{11,14}$. Flavio Nobili ${ }^{12,13,14}$
}

Received: 15 February 2019 / Accepted: 20 March 2019 / Published online: 9 April 2019

(c) Italian Association of Nuclear Medicine and Molecular Imaging 2019

\begin{abstract}
Introduction Recommendations about clinical and pathologic diagnosis of dementia with Lewy bodies (DLB) have been recently refined by the DLB Consortium. Substantial new information has been incorporated with increased diagnostic weighting given to molecular imaging biomarkers. The present work attempted to present a comprehensive evaluation of the role of molecular imaging in the frame of the revised DLB criteria.

Methods To this end, we briefly review the molecular imaging tools in the fourth Consensus report of the DLB Consortium, highlighting several indicative and supportive surrogate markers, including I-123 brain dopamine transporter (DaT), I-123 mIBG cardiac norepinephrine transporter (NeT) and brain F-18 fluorodeoxyglucose (FDG) imaging, as the main way to increase accuracy of ante-mortem diagnosis of probable or possible DLB.

Results Along with main neuropathological and clinical issues, we focus on the diagnostic performance and appropriate use of current available items included in the index by nuclear medicine physicians, namely a low DaT uptake, a low NeT expression in myocardial tissue, and reduced parieto-occipital metabolism on brain FDG-PET. Moreover, a critical summary of the current state of the art in pathological validation of other biomarkers including amyloid and tau-PET imaging is provided.

Discussion DLB Consortium clearly states that clinical diagnosis in clinical routine is suboptimal and gives more weight to molecular imaging biomarkers to offer a more objective information. Along with DaT, mIBG and FDG techniques, brain PET with more specific radiotracers could open a new scenario for an accurate evaluation of biomarkers involved in DLB.
\end{abstract}

Keywords DLB $\cdot$ Molecular imaging $\cdot$ DLB criteria $\cdot$ MIBG imaging $\cdot$ DaT imaging $\cdot$ FDG-PET

Stelvio Sestini

stelvio.sestini@uslcentro.toscana.it

1 Nuclear Medicine Unit, Nuovo Ospedale di Prato (NOP) S. Stefano, Prato, Italy

2 Nuclear Medicine Unit, San Raffaele G. Giglio Institute, Cefalù, Palermo, Italy

3 Nuclear Medicine Unit, Department of Biomedical, Experimental and Clinical Sciences, University of Florence, Florence, Italy

4 Department of Diagnostic Imaging, Radiation Oncology and Haematology, Institute of Nuclear Medicine, Fondazione Policlinico Universitario A. Gemelli IRCCS, Università Cattolica DEL Sacro Cuore, Rome, Italy

5 Nuclear Medicine Unit, Department of Medicine-DIMED, Padova University Hospital, Padua, Italy

6 Department of Biomedicine and Prevention, University Tor Vergata, Rome, Italy
7 Genoa Section, National Institute of Nuclear Physics (INFN), Genoa, Italy

8 Positron Emission Tomography Centre, IRMET S.p.A., Affidea, Turin, Italy

9 Department of Nuclear Medicine, Poliambulanza Foundation, Brescia, Italy

10 Institute of Biostructure and Bioimaging, National Research Council, Naples, Italy

11 Fondazione IRCCS Istituto Neurologico Carlo Besta, Milan, Italy

12 Department of Neuroscience (DINOGMI), University of Genoa, Genoa, Italy

13 IRCCS Ospedale Policlinico San Martino, Genoa, Italy

14 IRCCS Neuromed, Pozzilli, IT, Italy

15 Padova Neuroscience Center, University of Padua, Padua, Italy 


\section{Introduction}

It has been shown that dementia should not be considered as a "disease" but rather as a "syndrome" composed of signs and symptoms that can be caused by multiple diseases [1], one of which is the dementia with Lewy bodies (DLB) [2]. As well as for other dementias, such as Alzheimer's Disease (AD) [3], during the last decade the traditional leanings of incorporating biomarkers into models of DLB began considering the "clinical core features" as main markers of the disease, and worked backward to relate such clinical features to "pathological findings from autoptic studies" to sustain such choice [1, 4]. However, increasing evidence clearly showed that the approach of moving mainly from the clinical features as markers of disease presented with several limitations [5]. Indeed, clinical core symptoms were reported to appear relatively late in the course of the disease and to overlap with clinical symptoms of other dementing diseases, especially in the earlier stage, thus making difficult to distinguish DLB from other neurodegenerative dementias based on clinical manifestations alone [6]. On the other hand, results of epidemiologic and clinical studies showed that DLB is the second commonest cause of neurodegenerative dementia, and that it progresses more rapidly than other dementing disorders, harboring a poor prognosis due to severe cognitive impairment and parkinsonism [7]. For these reasons, the scientific community decided to renew the efforts to find out a way for a more accurate and early diagnosis. A subtle different approach was increasingly attempted where the pathophysiological changes detected by in vivo molecular imaging biomarkers may help defining the disease along with clinical core feature even in the early stage $[1,8]$. Classifying DLB by in vivo biomarkers, indicative of pathophysiological changes, together with clinical core features, represents a profound shift in thinking. For many years, DLB was conceived only as a clinical-pathological construct where symptoms/signs defined the presence of the disease in living persons, and, therefore, the concepts of symptoms and disease became interchangeable [4]. Later on, DLB has become mainly a clinical-biomarker construct, where in vivo pathophysiological biomarkers, including molecular imaging, presented once again with limited diagnostic weight [9]. More recently, although the definition of DLB was not divorced from clinical symptoms, in vivo pathological biomarkers were more heavily used to support a diagnosis of DLB in symptomatic individuals. Indeed, to increase the accuracy of antemortem diagnosis, the DLB Consortium has refined its recommendations, updating the previous report, which has been in widespread use during the last decade [10]. Substantial new information has been incorporated about previously reported aspects of DLB, with increased diagnostic weighting given to molecular imaging biomarkers.

In the present paper, recommendations included in the fourth DLB Consortium consensus report have been discussed and their implications highlighted, emphasizing the role of molecular imaging in defining DLB on a biological ground. The diagnostic performance and appropriate use of current available items included in the index used by nuclear medicine physicians for the diagnosis of DLB, namely a low dopamine transporter (DaT) uptake in the basal ganglia, a low norepinephrine transporter $(\mathrm{NeT})$ in sympathetic myocardial tissue and reduced occipital metabolism, have been discussed along with neuropathological and clinical concerns. The potential role of other biomarkers, including amyloid and tau-PET imaging to be recognized as diagnostic tools of DLB, has been also evaluated.

\section{Neuropathology}

DLB is pathologically characterized by Lewy bodies (LBs) and Lewy neurites (LNs) in the brainstem, limbic system, and neocortical areas. Classic LBs are intra-cytoplasmic eosinophilic neuronal inclusions that, in the pigmented brainstem neurons of substantia nigra and locus coeruleus, typically appear as dense, hyaline eosinophilic cores surrounded by a less densely stained halo. Unlike classic brainstem, cortical LBs are not easily seen in routine hematoxylin and eosin (H\&E) stain, appearing as ill-defined, pale, slightly eosinophilic inclusions in the perikarya of layer $\mathrm{V}$ and VI pyramidal neurons. For this reason, cortical LBs have long been underreported until the advent of more modern techniques (anti-ubiquitin and, more recently, anti$\alpha$-synuclein immunostaining) facilitating their identification. LNs represent a neuro-filament abnormality that is invisible using conventional H-E staining but is easily detectable by anti-ubiquitin or anti- $\alpha$-synuclein immunostaining. They typically occur in the CA2-CA3 of the hippocampal formation, the amygdala, the nucleus basalis of Meynert, the dorsal vagal nucleus and other brainstem nuclei, as well as in the peripheral autonomic nervous system. The main component of LBs and LNs is an anomalous $\alpha$-synuclein, which is phosphorylated, nitrated and truncated, has abnormal solubility, prompts the production of oligomeric species, aggregates into fibrils, and is ubiquitinated [11]. Although the presence of subcortical and cortical LBs is the only requisite for a pathologic diagnosis of DLB, further pathological concomitants can be LNs, regional neuronal loss-especially in the brainstem (substantia nigra and locus coeruleus) and in the forebrain (nucleus basalis of Meynert), microvacuolation (neuropil spongiform changes, not immunoreactive to prion protein), and $\mathrm{AD}$ changes. In this respect, while $\beta$-amyloid deposits in the form of diffuse and neuritic neocortical plaques are common and usually so numerous to fulfill both 
NIA and CERAD criteria for AD (the Lewy body variant of Alzheimer disease, LBV), neocortical neurofibrillary tangles (NFT) are rare, occurring in no more than one-fifth of the cases. Furthermore, although neuritic plaques of both DLB and $\mathrm{AD}$ brains have thickened silver-positive dystrophic neurites surrounding an amyloid core, those of DLB brains do not usually contain AT8 immunoreactive hyperphosphorilated tau protein. Of note, only a minority of DLB subjects have no or negligible AD pathology (pure DLB) [12]. DLB and Parkinson's disease (PD) with superimposed dementia (PDD) share similar pathologic characteristics. As a result, in the absence of clinical details, the pathologist who examines a brain with the aforementioned abnormalities, can hardly or not at all discern what was the disease in question, whether DLB or PDD. The distinction between these two entities may sometimes be not so easy even when the diagnosis is based on clinical rather than pathologic grounds because, according to Consensus Criteria [10], a clinical diagnosis of DLB can apply not only to cases with dementia preceding the onset of parkinsonism, but also to cases with parkinsonism occurring concurrently or within 1 year before the onset of dementia (so called 'one-year rule'). However, looking at groups rather than individual subjects, there is much greater concomitance of beta-amyloid deposits and neuritic plaques in DLB than PDD (probable or definite AD according to CERAD criteria, $87 \%$ vs $42 \%$ ) [13]. Furthermore, compared to PDD, DLB subjects show less neuronal loss in the substantia nigra pars compacta, which explains their less severe parkinsonism, and less postsynaptic D2 receptor upregulation, which explains their greater susceptibility to side effects of antipsychotics. As for plaques in subjects with no cognitive impairment ("preclinical AD"), LBs have been observed in the substantia nigra and cerebral cortex of subjects who have never exhibited clinical parkinsonism or cognitive deterioration during life (incidental LB disease, ILBD). LNs in the peripheral autonomic nervous system are usually observed early in these neurologically unimpaired subjects but, relative to subjects with full-blown disease, more brain areas can be spared by LBs/LNs and less numerous LBs/LNs can be found in the brain areas involved. Additionally, tyrosine hydroxylase immunoreactivity in the striatum and epicardial nerve fibers is lower in subjects with incidental LBs than in those without (normal controls), but this reduction is not as much as that observed in subjects with PD, suggesting that ILBD is a preclinical form of LB disease and the absence of motor and cognitive symptoms is attributable to subthreshold pathology.

Cortical LB density has been correlated with severity of cognitive impairment, but whether the amount of $\alpha$-synuclein pathology (i.e., LBs and LNs) in the cerebral cortex indeed is the major correlate of dementia in DLB is still a matter of controversy. Cholinergic and dopaminergic denervation of the neocortex also contributes to cognitive deficits in DLB [14], but deficits in neurotransmission are not restricted to dopaminergic and cholinergic systems. Synaptic damage, likely related to toxic $\alpha$-synuclein oligomers and pore formation, occurs in the neocortex of DLB subjects, and is accompanied by abnormalities in neurotransmitter signaling in a way similar to that reported for other $\alpha$-synucleinopathies. Additional molecular alterations converge in the pathogenesis of DLB, including impaired autophagy and ubiquitin-proteasome system of protein, as well as altered responses to protein misfolding. Preliminary studies have also shown that inflammation and oxidative damage, involving protein, lipids, and DNA, as well as impairment in mitochondrial activity, in energy metabolism, in purine metabolism, and in protein synthesis may be important factors in the pathogenesis of DLB.

Vascular pathology, including amyloid angiopathy, has also been demonstrated to be associated with DLB, contributing to severity of cognitive impairment [15].

Regarding evolution and distribution of pathologic alterations in diseases with LBs, an $\alpha$-synuclein staging scheme has been proposed by Braak and colleagues, who hypothesized that $\alpha$-synuclein in the form of Lewy body and neuriterelated pathology (LRP) first appears in the enteric nervous system, dorsal motor nucleus of the vagus nerve and the olfactory bulb [16]. However, this staging system is less suitable to DLB than PD. Its failure to classify a significant number of DLB cases has led to continuing debates about where LRP first appears and how it progresses, prompting alternative $\alpha$-synuclein staging systems to be proposed for LRP propagation in DLB (see below).

In the 2005 revised diagnostic criteria for DLB [9], cases were stratified into brainstem-predominant, limbic and diffuse neocortical types, according to the extension and diffusion of LRP. Since previously proposed by Japanese investigators, this classification was not novel, but it was recognized for the first time that the likelihood of occurrence of the typical clinical DLB syndrome was positively related to the extent of Lewy body pathology (diffuse neocortical $>$ limbic predominant $>$ brainstem) and negatively related to the presence/severity of Alzheimer neurofibrillary pathology, explaining why several DLB cases can clinically be missed and mostly diagnosed as having pure AD. Even increased neuritic plaque density makes the typical DLB syndrome less likely to occur, while beta-amyloid load has no effect [17] (Table 1). It may be that the "masked" clinical phenotype of DLB cases with coincident AD pathology is attributable to a different pattern of LRP propagation and distribution than that of pure DLB cases [18] and, in particular, to a negligible involvement of brainstem (thereby explaining the absence of parkinsonism) and extracranial districts (autonomic nerves) (Table 2). Of note, because cases with LRP restricted to the amygdala are common in both familiar and sporadic AD, and are unlikely to express 
Table 1 Assessment of the likelihood that the pathologic findings are associated with a DLB clinical syndrome-Alzheimer neurofibrillary/neuritic type pathology

$\begin{array}{lll}\text { NIA-Reagan low (Braak stage } & \text { NIA-Reagan intermediate (Braak stage } & \text { NIA-Reagan high (Braak stage V- } \\ \text { 0-II) CERAD neuritic plaque } & \text { III-IV) CERAD neuritic plaque score A } & \text { VI) CERAD neuritic plaque score } \\ \text { score } 0 & & \text { B-C }\end{array}$

\begin{tabular}{llll}
\hline Lewy body-type pathology & & & \\
Brainstem-predominant & Low & Low & Low \\
Limbic (transitional) & High & Intermediate & Low \\
Diffuse neocortical & High & High & Intermediate \\
\hline
\end{tabular}

Beta-amyloid load has no effect on the likelihood of occurrence of the DLB clinical syndrome

$D L B$ dementia with Lewy bodies, NIA National Institute on Aging; CERAD neuritic plaque scores: $O$ no neuritic plaques, $A$ sparse neuritic plaques, $B$ moderate neuritic plaques; $C$ frequent neuritic plaques

Table 2 Scheme of hypothetical progression pathway for Lewy body diseases

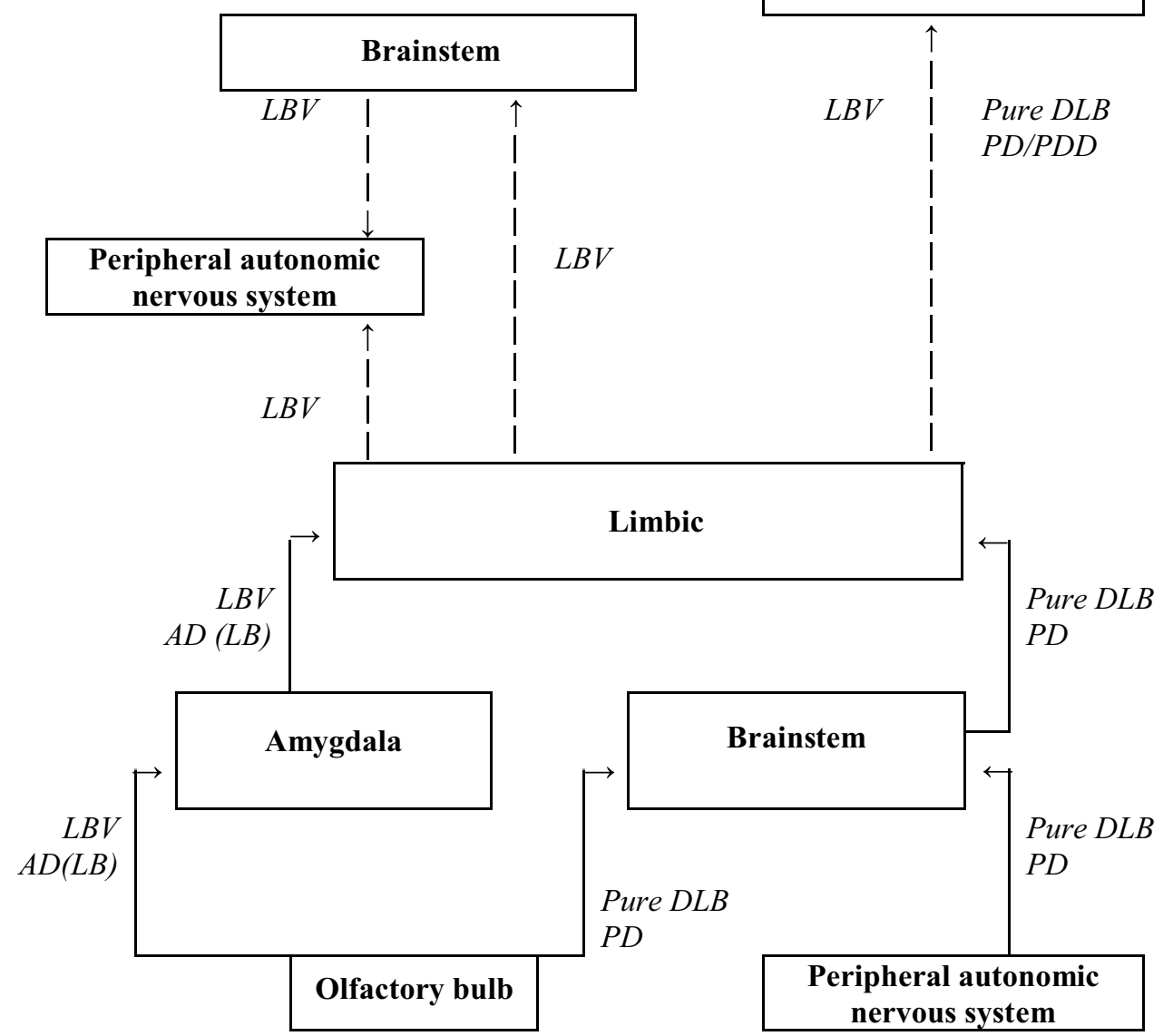

$L B V$ Lewy body variant of Alzheimer disease (common form of Dementia with Lewy bodies, pure DLB pure dementia with Lewy bodies (less usual form of Dementia with Lewy bodies), $P D$ Parkinson disease, $A D(L B)$ Alzheimer disease and Lewy bodies (but insufficient to meet criteria for DLB) the core features typical of DLB, they are not labeled as DLB by pathologists.

Functional imaging using brain PET with more specific radiotracers could open a new scenario for an accurate evaluation of biomarkers involved in neurodegenerative diseases, including DLB. Mirroring the findings of pathologic studies which, as noted above, show the common concomitance of AD plaque changes, PET with amyloid tracers is often positive in subjects with DLB. Studies with tau tracers are so far much fewer but, in light of pathologic studies showing only occasional presence of neurofibrillary tangles in the neocortex ( $20 \%$ of the cases), a negative neocortical uptake should 
contribute to favor a diagnosis of DLB over AD. Conversely, a positive neocortical uptake is not necessarily more in favor of an AD than a DLB diagnosis, as shown in a recent study [19] in which $\left[{ }^{18} \mathrm{~F}\right]-\mathrm{AV}-1451$ uptake was observed in the inferior temporal gyrus and precuneus of many DLB subjects. Interestingly, these cortical tau aggregates were associated with severity of cognitive impairment and were present even in those DLB subjects without elevated amyloid levels.

\section{Clinical hallmarks}

The clinical diagnosis of DLB can be a challenge in the memory clinics, particularly in small clinical centers where the most recent diagnostic techniques have not been implemented yet [20]. This derives from the overlap of some signs and symptoms with those of other dementing conditions, including mainly $\mathrm{AD}$, frontotemporal lobe degeneration (FTLD), and vascular cognitive impairment (VCI). Indeed, clinical tools (including neurological examination and neuropsychological assessment) are poorly sensitive ranging from 62 to $50 \%$ when evaluating a mixed pool of patients with DLB, AD or PD [21, 22]. A "wait and see" approach cannot be accepted for diagnosis confirmation and in the last 2 decades the diagnostic criteria have been repeatedly revised, including both clinical signs/symptoms and biomarkers.

The 2017 criteria [10] have introduced relevant novelties among categories and core symptoms of DLB. Indeed, if a patient with dementia presents two clinical core features, then the diagnosis is probable DLB, irrespective of biomarker availability. If instead the patient presents only one clinical core feature, then at least one positive biomarker is required among indicative ones to allow a diagnosis of probable DLB. Possible DLB is defined by an isolated clinical core feature or an isolated indicative biomarker. In no case, the diagnosis of probable DLB can be made based on biomarkers only. Besides, among the classical clinical core symptoms a new entry has been added: the REM sleep behavior disorder (RBD), highly specific for alphasynucleinopathies, i.e., DLB, PDD, and multisystem atrophy (MSA) [23, 24]. However, it can be ascertained only by means of polysomnography (PSG) that is costly and not available in all centers. Instead, 'probable' RBD is defined on clinical grounds. Its detection is increased using ad-hoc questionnaires, such as the Mayo sleep questionnaire [25] that has been PSG-validated and can be easily administered in a clinical setting. Briefly, what happens is that the physiological muscular atony during the REM sleep stage, that prevents the subject moves during dreams, is partially lost in DLB patients that 'act' their dreams. This can result in harmful behavior both to the patients and to the bed partner.

Other clinical signs/symptoms in the patient history or physical examination may be helpful and should be carefully checked; they are listed among the 'supportive' clinical symptoms. Far from being of 'limited' relevance, they are often almost important as the 'core' symptoms, especially when they are present in combination and the core features are few or uncertain. Severe sensitivity to neuroleptics [26], orthostatic hypotension, constipation and hyposmia are not specific for DLB but when they are both found together with other symptoms can be of great help to address the diagnosis. Hyposmia should be measured using tests available on the market $[27,28]$ and not merely asked for, because it has been shown that the report of patients and relatives is often inaccurate.

Other signs/symptoms can be checked in the McKeith et al. [10] paper and are summarized in Table 3. If only one core symptom is present then there is need of a positive

Table 3 Key points for the nuclear medicine physician in clinical routine setting

Patient with cognitive impairment and at least one of the following items

Structured VH referred by the relatives and often uncriticized by the patient

Mainly axial hypokinetic-rigid, symmetric spontaneous parkinsonism Drop of attention during the clinical interview; circadian fluctuations referred by the relatives

Bed-partner referral of patient 'acting' his/her dreams, shouting, falling from the bed while sleeping
Simple signs/symptoms that can be assessed and reinforce the clinical suspect of DLB

Measure blood pressure in sitting position and then after 3 min of standing: a drop $>20 \mathrm{mmHg}$ in systolic $\mathrm{BP}$ and $>10 \mathrm{mmHg}$ in diastolic BP stands for $\mathrm{OH}$ (consider that anti-hypertensive agents and diabetes can cause $\mathrm{OH}$ )

Ask the patient and the relatives if he/she has impaired smell; test it, if possible, with a standardized test, otherwise with home-made measures (coffee, alcohol, perfume...)

Ask the patient if he/she suffer from chronic constipation and/or urinary incontinence

Ask the relatives if there are hallucinations other than $\mathrm{VH}$, mainly auditory hallucinations

Investigate whether the patient falls during the past year

investigate whether the patient has become very rigid after receiving neuroleptics (typically haloperidol) to control hallucinations or agitation

Try to understand whether the patient is depressed, apathetic or anxious 
indicative biomarker. Clinical differential diagnosis includes mainly AD, PD, VCI, and behavioral variant FTLD (bvFTLD). Just to summarize the most common situations, visual hallucinations $(\mathrm{VH})$ and parkinsonian signs may be expressed by $\mathrm{AD}$ patients, often in a more advanced stage than in DLB, but substantial overlap occurs. Parkinsonian signs can be found both in bv-FTLD and VCI, and attention fluctuations in VCI. Fluctuations deserve an ad-hoc comment as they can be appreciated by an expert but may be difficult to assess by a less experienced physician. For this purpose, the interview with an informant is of great help in identifying periods during the day of sleepiness, drowsiness, and motor stops, better if quantified through a formal and simple scale, such as the Clinicians Assessment of Fluctuation [29].

As for neuropsychological characteristics, it is mandatory that a patient with suspected DLB and mild dementia undergoes a detailed neuropsychological test battery with special attention to visuospatial abilities and executive functions that are typically impaired. Also, verbal episodic memory, language and abstract reasoning must be assessed to guide the differential diagnosis with the other diseases. In moderate-to-severe dementia, detailed neuropsychological assessment is often unfeasible or simply unhelpful since all forms tend to overlap one another. However, even in neuropsychological tests some overlap exists with the other most common dementing conditions. Executive dysfunction is frequent also in VCI and bv-FTLD whereas visuospatial impairment becomes common also in AD as severity of dementia increase. Moreover, severe verbal memory impairment in delayed recall is typical of $\mathrm{AD}$, but may also present in DLB. This is why the clinical approach to the patient with DLB is so misleading and why there is often the need of diagnosis confirmation through biomarkers. Fortunately, MRI is of great help in defining VCI, and some behavioral disorders, such as disinhibition and voracity for sweets, are typical of bv-FTLD. Another issue is the overlap between DLB and PD. Both these diseases are LBs and alpha-synuclein pathologies and, as such, they share the majority of signs and symptoms. PD more often has an asymmetric motor presentation, sometimes with prevalent resting tremor, whereas parkinsonism in DLB is more often akinetic-rigid, axial, and symmetrical. VH are typical of DLB but can be present in PD dementia, and nowadays the presence of dementia is no longer an exclusion criterion for the diagnosis of PD at presentation [30]. At the earliest stages, it has been suggested that the two entities cannot be distinguished and more meaningfully we should talk about Lewy body disease (LBD), while the follow-up can clarify the clinical trajectories, if toward full-blown DLB or PD [31]. That said, when the clinical presentation is clear enough then the probability that a biomarker confirms the diagnosis is very high, that is, the clinical picture has a specificity approaching $90 \%$ [32].
On the other hand, DLB can still be present and the clinical picture be uncertain which is the most dangerous condition because if the clinician does not suspect DLB, neither he/ she will ask for biomarker confirmation. This is particularly true when the only core clinical symptom is not immediately evident, as in the case of RBD and attention fluctuation. In summary, the sensitivity of clinical diagnosis is as poor as about $60 \%$ [32].

A final but non-trivial issue is the definition of patients with a characteristic DLB presentation who are not demented yet, i.e., patients with prodromal DLB or Mild Cognitive impairment (MCI) due to LBD. Based on diagnostic criteria for DLB, dementia is by definition an essential requirement for DLB diagnosis, while criteria for prodromal DLB, albeit in preparation, are not yet available. This means that the diagnosis of DLB in pre-dementia stages can currently be hypothesized, but not made with the help of a formal support.

In summary, the diagnostic evaluation of a potential patient with DLB requires careful interview with the relatives, the partner (to ascertain RBD), the patient (to pick-up supportive symptoms such as constipation and hyposmia), and clinical examination to disclose $\mathrm{OH}$ and parkinsonism. If DLB is suspected, then we recommend diagnostic confirmation with indicative biomarker even when more than one core clinical feature is present, unless we are managing a very old and comorbid patient (i.e., older than 85). Confirming the diagnosis is crucial for prognostic purposes since DLB has generally a more aggressive course than $\mathrm{AD}$, PD and VCI, and similar to bv-FTLD, but even more to for therapeutic choices, such as acetylcholinesterase inhibitors, that have often good symptomatic effects while typical neuroleptics must be avoided (trazodone, quetiapine and clozapine being the most used drugs) [33].

\section{Imaging biomarkers of DLB}

The diagnostic biomarkers have been classified as indicative and supportive [10]. We will briefly describe, in this paragraph, the role of nuclear medicine indicative $\left({ }^{123}\right.$ I-Ioflupane as a marker of DaT, and ${ }^{123} \mathrm{I}$-metaiodobenzylguanidine, mIBG, cardiac uptake as a marker of the NeT) and supportive (FDG-PET) features.

\section{Dopamine transporter tracers}

The Society of Nuclear Medicine (SNM) practice guidelines for DaT imaging (dated 2011) and the European Association of Nuclear Medicine (EANM) procedure guidelines for brain neurotransmission SPECT (dated 2010) stated only

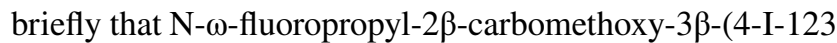
iodophenyl) nortropane ( ${ }^{123}$ I-Ioflupane, or ${ }^{123}$ I-FP-CIT) could be used as a support to differentiate DLB from other 
types of dementias, mainly $\mathrm{AD}$. On the other hand, results of imaging studies performed during the last 20 years have clearly highlighted the diagnostic potential of such molecular imaging tool to reveal in vivo degeneration of the nigrostriatal dopamine pathway in patients with DLB. Preliminary results of DaT imaging studies performed during the 90s up to results of those performed in the last 2 decades [34-48] have provided increasing evidence that its diagnostic accuracy is high enough to be clinically useful in distinguishing DLB from other types of dementias, especially AD. Indeed, a systematic meta-analysis on DLB diagnostic accuracy of presynaptic dopaminergic imaging with ${ }^{123}$ I-FP-CIT showed a diagnostic sensitivity of $86.5 \%$ and specificity of $93.6 \%$ in differentiating between subjects with DLB and AD [49]. Moreover, the Cochrane's review [50] suggested that such a diagnostic potential could be even higher if a semi-quantitative rating of ${ }^{123}$ I-FP-CIT SPECT scans instead of visual analysis is used. Accordingly, pooled results of imaging studies ( $150 \mathrm{pts})$ using semi-quantitative approach revealed that sensitivity can rise up to $100 \%$. The authors concluded that DaT imaging is more accurate than clinical diagnosis and that clinical diagnosis is unsuitable to be used as a reference standard for assessing the accuracy of DaT imaging for the diagnosis of DLB [50, 51].

However, uncertainty remains in the differential diagnosis between DLB and dementia types other than AD, especially the behavioral variant of frontotemporal dementia (bvFTD), and between DLB and either PD or other degenerative parkinsonisms. As regards to the differentiation between DLB and other dementia types, diagnostic accuracy has in fact been particularly high when the comparison with DLB patients was limited to AD patients with no or negligible parkinsonism, but a drop in specificity was noted when the non-DLB control group included or was restricted to patients with bvFTD having variable degrees of parkinsonism. With respect to the distinction between DLB and PD or atypical parkinsonisms, one of the most cited studies to this regard shows that DaT loss in DLB is of similar magnitude as in PD and that ${ }^{123}$ I-FP-CIT SPECT provided good separation between patients with a LB disease and AD (region of interest: sensitivity $78 \%$; specificity $94 \%$; positive predictive value $90 \%$ ) but not among subjects with DLB, PD, and PDD [52]. Furthermore, imaging results should be carefully related to clinical presentation because about $30 \%$ of patients with FTLD have shown a positive DaT imaging [53]. On the other hand, it should be noted that a negative DaT imaging will not exclude a DLB if the clinical presentation is consistent, because a small number of patients may disclose cortical and limbic pathology but not nigrostriatal involvement that eventually might follow after some time. Finally, we point out that when directly comparing ${ }^{18} \mathrm{~F}$-Fluorodopa $\left({ }^{18} \mathrm{~F}\right.$-DOPA) PET and ${ }^{123} \mathrm{I}$-FP-CIT SPECT, Eshuis et al. reported very similar accuracies [54].

\section{${ }^{123}$ I-mIBG myocardial uptake}

The mIBG radioligand is a non-invasive method to evaluate in vivo postganglionic presynaptic cardiac sympathetic innervation. Results of several studies have clearly demonstrated the diagnostic potential of mIBG imaging to detect in vivo the impairment of myocardial adrenergic system in patients with DLB [55-58]. For these reasons, myocardial mIBG imaging has been recently up-graded as an indicative biomarker of DLB [10]. Indeed, in about 2680 subjects from 46 studies, King AE et al. demonstrated $94 \%$ sensitivity and $91 \%$ specificity for the diagnosis of LB-related disorders, including DLB [59]. Concerning the role of $\mathrm{mIBG}$ in clinical setting with respect to DaT imaging, the former has been firstly suggested as an alternative to FP-CIT SPECT in the diagnosis of DLB as compared to other dementias [60]. More recently, results of imaging studies evaluating the usefulness of DaT-SPECT and mIBG cardiac scintigraphy in patients with suspected DLB have suggested a complementary role of the two tools [61]. In one study, it has been shown that DaT-SPECT differentiates DLB from AD better than mIBG scintigraphy, due to its higher sensitivity. On the other hand, mIBG scintigraphy seemed to exclude DLB better than DaT-SPECT, due to its higher specificity [62, 63]. When parkinsonism is the only clinical "core feature", it has been shown that mIBG imaging is more specific than DaT-SPECT for excluding non-DLB dementias [64, 65]. For instance, a recent study evaluating the role of DaTSPECT and mIBG cardiac scintigraphy in uncertain parkinsonian conditions associated to cerebrovascular lesions has shown that when vascular lesions in striatal nuclei and in white matter occur, DaT-SPECT alone is not able to discriminate between vascular and degenerative parkinsonism, while it is possible to achieve the most appropriate diagnosis using mIBG cardiac scintigraphy [61]. Importantly, it has been shown that cardiac sympathetic function in DLB is severely impaired even in the early stages [66] and that mIBG scintigraphy is able to reveal such dysfunction [67-70]. With this respect, it is to highlight that mIBG scintigraphy should be employed using a semi-quantitative approach. Indeed, semi-quantitative measurements of myocardial mIBG uptake can be derived including early and late heart-to-mediastinum $(\mathrm{H} / \mathrm{M})$ ratio (HMR) and mIBG washout. Manually defined regions of interest (ROIs) on planar images are conventionally used to compute myocardial uptake parameters [71] while HMR reflects the mIBG uptake in nerve terminals, washout rate indicates their integrity-neuronal retention. The diagnosis of DLB has been shown to be associated with a significant decrease of $\mathrm{H} / \mathrm{M}$ ratio compared with that of $\mathrm{AD}$ or parkinsonism including $\mathrm{PD}$, especially using the late 
images. Nevertheless, it is to be noted that the lack of a shared quantification procedure causes differences in cutoff values among centers [72], as $\mathrm{H} / \mathrm{M}$ ratios are mostly determined on ROIs that may differ in size and shape and by the use of different collimators (low or medium energy), explaining the cutoff variability reported in the literature. Furthermore, it has also been shown that the use of a two-dimensional planar imaging instead of SPECT acquisition protocol presents further potential limitations that may decrease the accuracy of procedure including the impossibility to disentangle the un-specific lung uptake from the specific cardiac uptake. With this respect, it has been shown that SPECT/CT hybrid systems may have the potential to improve ROI localization and thus the accuracy of mIBG semi-quantitative scintigraphy [73]. Finally, values of $\mathrm{H} / \mathrm{M}$ ratios may decrease during pathological (e.g., various cardiovascular morbidities, latent cardiac disorder and medications, which may damage the postganglionic sympathetic neurons) or physiological conditions (e.g., age and show gender-specific variations), possibly leading to false-positive findings [74].

\section{F-FDG-PET}

FDG-PET was reported to be useful to distinguish DLB from AD by showing a severe reduction in glucose metabolism in the occipital cortex, besides variable degrees of hypometabolism in parieto-temporal and frontal association cortex which may be shared by AD as well [75]. Indeed, involvement of association occipital cortex is evident mainly in DLB patients. The occipital hypometabolism could be associated with visual cortex neuropathology, as reported in a small autopsy-confirmed sample of DLB patients [76, 77]. The authors reported several findings including: (1) a metabolic ratio of 0.92 in the visual associative cortex toward whole brain uptake allowed to distinguish DLB from AD with very high sensitivity and specificity; (2) the regional metabolic changes in each brain region generally paralleled the severity of the spongiform changes; and (3) the occipital hypometabolism preceded some clinical features, reflecting the worse visuospatial and visual-constructive deficits. The reason for occipital hypometabolism is not clear and two hypotheses have been postulated: (1) dopaminergic abnormalities in the visual pathway before the occipital lobe terminal; and (2) lower activity of choline acetyltransferase secondary to the neurodegenerative processes in the basal nucleus of Meynert or other cholinergic nuclei in the brainstem, preferentially involving neurons projecting to the occipital lobe [78]. This selective deafferentation may contribute to the occipital hypometabolism suggesting that it is not related to brain tissue loss (a hypothesis supported by the lack of atrophy at MR) but to disruption of intra-cortical connections [79]. Another characteristic sign of DLB is the relatively preserved glucose metabolism in the posterior cingulate area when compared to precuneus and cuneus, the so called "cingulate island sign" (CIS), as reported in FDG-PET semi-quantitative analysis $[78,80]$. This sign has a higher specificity (100\%) compared to occipital hypometabolism, could be related to visual hallucinations, improves with acetylcholinesterase inhibitor treatment, and correlates inversely with neurofibrillary tangle pathology [80, 81]. The CIS was not associated with amyloid-ß (Aß) load on Pittsburgh compound $\mathrm{B}$ (PiB) PET and is thought to be related to impaired cholinergic transmission or synaptic dysfunction associated with alpha-synuclein; in addition, the CIS predicts a lower Braak neurofibrillary tangle stage [81]. A large number of DLB patients have, however, coexisting AD pathology. The recognition of the degree of $\mathrm{AD}$ pathology in DLB patients is important because imaging biomarkers of coexisting AD pathology predict a worse cognitive decline $[82,83]$, treatment response to acetylcholinesterase inhibitors in DLB patients and may also be a predictor of poor survival [81]. Therefore, the CIS on FDG-PET may be used in the differential diagnosis of DLB while Aß-PET might give more information on prognosis. However, despite the preliminary results of FDG-PET seems promising, there is not yet a proven enough accuracy for it be considered as an indicative biomarker in the new criteria $[4,10]$.

\section{Other imaging biomarkers}

Alpha-synuclein deposition in LBs is the core neuropathological feature of DLB but A $\beta$ and tau co-pathologies are commonly reported in most patients' post-mortem. The successful development of PET radioligands to image fibrillar $A \beta$ has allowed to investigate in vivo cerebral amyloidopathy in DLB while the recent development of PET tracers selective for tau, although much more complex and still at an exploratory research stage, provided the preliminary results of tau load in DLB. No suitable PET tracers for alphasynuclein imaging is currently available but new tracers are emerging that might be tested in future studies.

\section{Amyloid imaging}

Cerebral amyloid burden in DLB has been widely studied in vivo with PET during the last 10 years, mostly using ${ }^{11} \mathrm{C}$-PIB. Increased cortical ${ }^{11} \mathrm{C}$-PIB uptake was commonly reported in DLB patients as compared to PDD, PD (independently of their cognitive status), and controls in the similar range or slightly lower than in AD [84]. Elevated cortical ${ }^{11} \mathrm{C}$-PIB retention in DLB ranged between $50 \%$ and $100 \%$ of patients in most part of studies although in some reports it was less frequently observed (20-44\%) [19, 85]. Although in PDD, $\mathrm{A} \beta$ accumulation is less frequent and milder than 
in DLB, a finding supported by post-mortem data (44), increased cortical amyloid binding in the range of DLB and AD has been reported in a minority of PDD patients [86], thus limiting the possibility of differentiating the two groups. In non-demented PD patients, the rate of positive amyloid scans is usually low although longitudinal studies suggested that the progression to cognitive impairment and dementia is faster in those with higher amyloid burden [86]. The regional distribution of increased ${ }^{11} \mathrm{C}$-PIB binding targeted cortical association areas, including the precuneus and cingulate, a pattern similar to that observed in $\mathrm{AD}$ [87] making it difficult to differentiate the two diseases. In some reports, however, occipital PIB retention was found to be higher in DLB as compared to $\mathrm{AD}$ [88] corresponding, in some cases, to reduced FDG uptake in the same areas [84], but these findings were not confirmed by others [89]. Comparable results were observed in few studies using ${ }^{18} \mathrm{~F}$-florbetaben $[84,90]$ or ${ }^{18} \mathrm{~F}$-Florbetapir [86]. In ${ }^{11} \mathrm{C}$-PIB-positive DLB and PDD patients, increased cortical ${ }^{11} \mathrm{C}$-PIB retention was associated to the presence of the APOE4 allele, lower MMSE scores, and low levels of CSF A $\beta-42$ [87]. Moreover, a higher cortical amyloid burden in DLB may influence the timing of dementia onset with respect to motor symptoms [86]. In a recent review, a role of striatal amyloidopathy in cognitive impairment of DLB has been suggested while inconsistent correlation between amyloid deposition and cognitive functions was reported [91].

Overall, the results of the amyloid PET studies in DLB are heterogeneous and show a certain variability although increased cortical amyloid load is more frequently observed in DLB than PDD and cognitively affected PD and might contribute to dementia. The finding of relatively low amyloid rate in PD patients with cognitive impairment is intriguing and different from that observed in $\mathrm{AD}$ raising the question of different contribution of amyloid load in the future development of dementia in DLB and AD or possible protective role of alpha-synuclein.

The heterogeneity of neuropathological processes involved in DLB, the complexity of clinical classification of DLB and PDD that shared similar neuropathological and clinical findings, differences in patients's age and disease duration might explain only in part some discordant results. A recruitment bias has been also suggested to explain the wide range of positive amyloid scan observed at an individual level in DLB due to possibly higher inclusion of positive amyloid patients by dementia centers than movement disorders clinics [85]. The interpretation of these results is, however, hampered by other important limitations: (1) the small simple size of patients included and (2) the heterogeneity of methodologies used across studies. Most part of studies were limited to 3-14 DLB patients and only few studies included between 18 and 21 patients [85]. Concerning the methodologies, visual binary classification, static semi-quantitative
SUVR and dynamic quantitative DVR using mostly the cerebellum as reference region were differently used to provide an estimation of individual as well as mean changes in amyloid load. Although in most studies VOIs and voxel-based analysis were used for characterizing the regional pattern of increased amyloid load, in most studies a global cortical mean value was reported for assessing the significant increase of amyloid binding. In addition, these global cortical values were obtained by averaging different cortical regions and the majority of studies did not include occipital cortex, a region reported to be differently involved in DLB and $\mathrm{AD}$. Larger, prospective future homogeneous studies in terms of patient's inclusion and standardization of methods for data acquisition and analysis of spatio-temporal distribution of new fluorinated amyloid tracers are needed to clarify the role of amyloid in DLB with respect to differential diagnosis with AD and PDD and patient's classification for novel anti-amyloid interventions.

\section{Tau imaging}

The ${ }^{18} \mathrm{~F}$-flortaucipir, also named ${ }^{18} \mathrm{~F}-\mathrm{AV}-1451$, has been one among the most used tau-PET, although its specificity to Tau deposition is somewhat hampered by aspecific binding to other brain structures, such as the choroid plexus. In a preliminary study, both cortical ${ }^{18} \mathrm{~F}-\mathrm{AV}-1451$ uptake and ${ }^{11} \mathrm{C}$-PIB retention were studied in 7 DLB, 8 cognitively impaired PD and 9 cognitively unimpaired PD patients. Increased ${ }^{18} \mathrm{~F}-\mathrm{AV}-1451$ binding, lower in magnitude and extent than that observed in $\mathrm{AD}$, was found in DLB and PDcognitively impaired patients as compared to low-amyloid controls, although it was highly variable [19]. The topography of abnormal ${ }^{18} \mathrm{~F}-\mathrm{AV}-1451$ binding was similar to that observed in $\mathrm{AD}$ and was associated in the inferior temporal gyrus and precuneus with increased cognitive impairment. Interestingly, increased ${ }^{18} \mathrm{~F}-\mathrm{AV}-1451$ binding was not always associated with elevated amyloid retention differently from what observed in $\mathrm{AD}$ patients. In a subsequent study [92] performed in a larger group of patients (19 DLB) and including also $19 \mathrm{AD}$ and 95 controls, significant increased AV-1451 uptake was found in DLB as compared to controls whose magnitude was significantly lower than in AD group. Higher involvement of medial temporal cortex in AD and higher involvement of inferior and medial occipital cortex and sensory-motor cortex in DLB allowed differentiating the two diseases. Similarly, significantly elevated ${ }^{18} \mathrm{~F}$-AV1451 binding in the occipital, parieto-temporal and primary sensory-cortices was found but only in amyloid-positive DLB patients as compared to controls [90]. These findings are different from those of Gomperts et al. [19] and suggest that the ${ }^{18} \mathrm{~F}-\mathrm{AV}-1451$ binding in DLB has distinct patterns from $\mathrm{AD}$ and that amyloid burden plays an important role in neocortical tau accumulation. As for amyloid deposition in 
DLB, tau pathology detected using ${ }^{18} \mathrm{~F}-\mathrm{AV}-1451$ is uncommon in PD patients even when mild cognitive impairment is present [93]. Future studies in larger series of patients using more suitable and selective second-generation tau tracers are required to further confirm these data.

\section{Tracers for neuroinflammation}

Along with established milestones for functional imaging in DLB including radioligands for DaT or NeT transporters and glucose consumption, potential new targets have been investigated, such as neuroinflammation. Neuroinflammation is a multifunctional process involving complex pathways including oxidative stress and microglia activation. In response to insults it has been shown that microglia change from resting to an activated state potentially causing a cerebral damage due to an autoimmune reaction. For this reason, the in vivo detection of activated microglia may be used as surrogate marker of neural damage [94]. The most PET studies exploring neuroinflammatory processes involve the use of tracers binding specifically to a protein called TSPO, which is expressed on the activated microglia. The [N-methyl-11C](R)-1-(2-chlorophenyl)- $N$-(1-methyl-propyl)-3-isoquinolinecarboxamide (11C-PK11195) is the most commonly studied in vivo marker of neuroinflammation with a relative worthy cellular selectivity, allowing the measurement of increased TSPO with activated microglia and infiltrating macrophages with high affinity (human $\mathrm{Kd} 2 \mathrm{nM}$ ) [95]. Although the significance of neuroinflammation in DLB is still far to be completely known, the use of PK11195 in patients with DLB have shown an increased radioligand binding consistent with microglia activation possibly induced by $\alpha$-synuclein in brain regions known to be affected by disease. Beside, radioligand-binding potential was found to present a positive correlation with cognitive performance and a negative correlation with disease duration, suggesting that microglial activation could be elevated mainly in the early disease and that the potential immunotherapeutic window is narrow and early in DLB $[96,97]$. Even if this tracer could improve our knowledge in the pathophysiology of neuro-degeneration, its application in the clinical setting is hampered by several limitations, mostly related to the poor signal-to-noise ratio, the low permeability, ${ }^{11} \mathrm{C}$ labeling, the high level of nonspecific binding and genetic polymorphism in the TSPO affecting the binding affinity properties of most of PET TSPO radiopharmaceuticals. More recently, other tracers have been proposed including molecules able to label subtype 2 cyclooxygenase, metalloproteinases, cannabinoid receptor type 2 and nitric oxide synthase to identify whether the nature and extent of microglial activation in DLB can be linked to structural changes, progression of domain-specific cognitive symptoms and peripheral inflammation as a marker of central microglial pathology. Answer to these questions will enable the evaluation of immunotherapies as potential therapeutic options for prevention or treatment of DLB [94].

\section{Other biomarkers (RSWA, EEG, MRI, CSF, and genetics)}

RSWA is the neurophysiologic substrate of RBD, and it is especially useful to corroborate an RBD diagnosis especially when RBD mimickers (including nightmares, sleep terrors, nocturnal seizures, and obstructive sleep apnea) are suspected and need to be excluded. RSWA in the absence of overt RBD may, however, be present as an isolated, incidental finding during PSG performed for other reasons. This finding should prompt clinicians to obtain further information from the patient and his/her bed partner, since up to $50 \%$ of patients with idiopathic RBD may be unaware of their dream-enacting behavior. The significance and natural history of isolated RSWA needs to be further elucidated to clarify its role as a possible specific biomarker of underlying synucleinopathy. In this respect, it should be highlighted that RSWA can incidentally be found in patients taking antidepressant drugs and that even normal individuals may have short bursts of abnormal phasic or tonic muscle activity during REM sleep, an observation that led the American Academy of Sleep Medicine to suggest formal diagnostic standards for RSWA scoring during PSG [98]. Despite these caveats, there is already some evidence for isolated RSWA being considered a biomarker for underlying synucleinopathy, including greater conversion risk to idiopathic RBD and association with other possible biomarkers of prodromal LB disease, such as hyposmia. Isolated RSWA has also been reported in MCI patients without clinical RBD.

Among the advantages of electroencephalography (EEG), which indirectly measures synaptic integrity, are wide availability, lack of invasiveness, and low cost. Although both visual and spectral analysis should be taken into account when interpreting EEG data, the latter is considered more specific. The main DLB features identifiable by visual EEG analysis are reduced reactivity of background activity and pronounced slow-wave paroxysmal activity, including frontal intermittent rhythmic delta activity. When analyzing EEG quantitatively (qEEG), DLB has been associated with increased power in theta and delta frequency bands, a low dominant frequency and a high dominant frequency variability, with a diagnostic accuracy for DLB usually greater than $85 \%$ in studies comparing DLB with AD subjects [99]. The technique may also be useful in identifying DLB in its pre-dementia stage [100]. However, since the presence of the aforementioned abnormalities tightly correlates with fluctuations, whether DLB subjects with no or negligible fluctuating course exhibit such a distinctive EEG pattern remains less clear. 
MRI is commonly performed in demented patients and it is often the first available biomarker in clinical routine. Although its diagnostic utility is reported to be mainly related to its exclusionary role of secondary causes of dementia, MRI is also extensively used for evaluation of gray matter atrophy in several neurodegenerative dementias. In case of DLB, there is evidence that the estimate of severity of medial temporal lobe (MTL) atrophy and of cerebrovascular disease may serve to differentiate DLB from $\mathrm{AD}$ and vascular dementia, respectively [101]. Indeed, it has been shown that the topographic pattern of atrophy in DLB is usually characterized by global gray matter atrophy including temporal, parietal, frontal and insular cortices with a relatively specific preservation of the MTL $[101,102]$ and that such less MTL atrophy in DLB than AD subjects has a robust discriminatory power for distinguishing between these two types of dementia (sensitivity of $91 \%$ and specificity of $94 \%$ ) [101, 103]. For these reasons, the relative preservation of MTL volumes on MRI has been included as a biomarker supportive of clinical diagnosis in the recently revised criteria for DLB $[5,10,101]$. However, such a remarkable discrimination potential has been achieved with quantitative analysis tools. The accuracy of MRI in discriminating DLB from other types of dementias (especially AD) in clinical practice remains poor because it still relies on image visual analysis. With visual analysis, a sensitivity of $64 \%$ and a specificity of $82 \%$ have been reported in the distinction between $\mathrm{AD}$ and DLB while higher values have been obtained in the differential diagnosis with frontotemporal dementia (FTD) with a sensitivity of $93 \%$ and a specificity of $89 \%$ [104]. Furthermore, along with pure DLB, results of autopsy-confirmed cohorts have shown that MTL atrophy can be found in DLB subjects but only if a concomitant AD-type pathology is present [104]. Besides, results of this study have also shown that such AD-variant of DLB is especially associated with neurofibrillary tangle density [105]. Importantly, compared to pure DLB, these mixed DLB/AD patients display a faster progression of cognitive impairment and higher rates of global atrophy over time [106]. Mirroring such findings, the presence of AD biomarkers in the cerebrospinal fluid (CSF) of DLB subjects, especially reduced levels of $A \beta$-amyloid 1-42, has been associated with greater cognitive decline, reaffirming the prognostic significance of concomitant AD-type pathology in DLB [107]. CSF alphasynuclein levels are decreased in DLB compared with AD and controls [108] but, due to overlap across groups, this reduction is not diagnostically useful in individual cases. CSF alpha-synuclein oligomers are by contrast increased in DLB compared with AD and controls [109] and apparently with less overlap across groups than with alpha-synuclein, but its diagnostic value needs to be further elucidated.

As a perspective, the search for a pathological biomarker in the DLB field has so far provided controversial results.
An interesting approach is to concurrently consider multiple peripheral sites to identify the best biofluids and tissues for measuring alpha-synuclein outside of the brain. One of the most promising sites appears to be the skin, where alpha-synuclein deposits may be found in the peripheral nerve fibers of the autonomic nervous system. Using cervical skin biopsy, Donadio et al. [110] obtained a sensitivity and specificity of $100 \%$ for phosphorylated alpha-synuclein deposits in DLB patients. However, in this study, the AD control group was much younger than usual (and significantly younger than the DLB group). More numerous and representative control samples, especially with $\mathrm{AD}$, are, therefore, required to confirm these impressive observations.

Regarding genetic biomarkers, although most DLB cases seem to be sporadic, rare autosomal dominant inheritance has been reported, including mutations in the $\alpha$-synuclein gene (SNCA). A significant proportion of DLB patients carry pathogenic mutations or risk variants in glucocerebrosidase (GBA) and Apolipoprotein E (ApoE) genes, suggesting that genetic defects may play a role in pathogenesis and prognosis of DLB. Besides confirming these associations, a recent study has provided some evidence for a novel locus, namely CNTN1, a glycosylphosphotidylinositol—anchored neuronal membrane protein that may serve as a cell-adhesion molecule [111]. However, the relatively unclear role of genetic variability and genetic defects in the pathogenic process limits their application in clinical practice, which remains elusive.

\section{Probable and possible DLB criteria: avoiding biomarkers replicating clinical feature}

If the clinician still has doubts about the diagnosis or needs further confirmation of his/her construct after careful clinical and neuropsychological evaluation, the use of available biomarkers requires some reasoning based on the peculiar features of each of them, their advantages and limitations, availability, and costs. Starting from indicative biomarkers, mIBG cardiac scintigraphy and DaT-SPECT or DOPAPET share similar diagnosis accuracy but actually explore different pathophysiological markers, i.e., the impairment of noradrenergic cardiac system and of the dopaminergic nigrostriatal system, respectively. The mIBG scintigraphy cannot be chosen in the presence of severe cardiomyopathy or uncontrolled, long-lasting diabetes, but it may still be an option in milder stages of these comorbidities, when the interference with mIBG uptake may still be negligible $[64,112]$. More controversial is the medication effect for inhibition of mIBG uptake, but it is likely that a smaller number of compounds than formerly believed can do it significantly. A sufficient evidence to recommend withholding prior to mIBG imaging has in fact been reached for only labetalol and tricyclic antidepressants [113]. A substantial 
part of patients with an impaired mIBG scintigraphy does not show symptoms or signs of autonomic failure, meaning that the procedure is more sensitive than the corresponding clinical symptoms/signs. Taking this reasoning into account, mIBG scintigraphy could be redundant if, instead, a patient with suspected DLB already presents, in addition to orthostatic hypotension, clear signs of autonomic failure, such as impotence and/or incontinence. In these instances, the choice of the indicative biomarker should be directed toward the other two. On the other hand, DaT imaging explores a function that is correlated with a core clinical feature, i.e., parkinsonism. It could be redundant using $\mathrm{DaT}$ imaging to confirm diagnosis in a patient whose only clinical core feature is Parkinsonism, or even if the patient has parkinsonism plus another clinical core feature [114]. Also, a patient with clear neuroleptic sensitivity has a high pre-test probability of having an altered DaT imaging, and another indicative biomarker might be chosen. In these instances, using another indicative biomarker may be recommended. Despite these intuitive reasons, there is no approved or shared recommendation on which indicative biomarker to use, according to the presence of specific clinical core features of the disease. Moreover, there are few studies comparing more biomarkers in the same group $[64,115]$. As for PSG, it is certainly of great utility but its availability is limited to the main clinical centers. Also for PSG the same reasoning as for the other two main biomarkers can be applied, i.e., it might be less useful if the clinical interview and a questionnaire for the presence of RBD are already clearly positive, while it can be of great utility if RBD does not appear in the clinical history. In summary, we suggest using an indicative biomarker unrelated to the corresponding clinical feature, to overcome circularity.

Another consideration is deserved to FDG-PET and EEG. Especially for FDG-PET, its clinical use is increasing greatly in patients with MCI of undetermined origin. The finding in such patients of the peculiar lateral occipital and precuneus hypometabolism with relative preservation of metabolic levels in the posterior cingulate may prompt further re-evaluation by the clinician and further confirmation with an indicative biomarker. In fact, DLB even in its prodromal stages has been recently included in the clinical recommendations on when to use FDG-PET [116]. EEG is less used in clinical routine for dementia patients but the finding of oscillations in alpha peak frequency as well as the abundance of delta waves in patient with MCI or mild dementia should prompt further re-evaluation, similarly as for FDG-PET findings. Finally, other non-scientific considerations should be taken into account in the choice of biomarkers. First, the costs and the local availability. Second, the local expertise in performing and reporting the result of these investigations. Finally, the availability of robust tools for quantification of data which is often the case for DaT imaging and FDG-PET but seldom for mIBG cardiac scintigraphy. Moreover, the latter is used off-label for this indication in some countries, including Italy.

\section{Concluding remarks}

Carefully examining criteria included in the fourth consensus report of the DLB Consortium gave us the possibility to highlight some considerations about the role of molecular imaging in the diagnosis and management of patients with DLB.

First, starting from the clinical point of view, the criteria refined by the DLB Consortium describe in detail and consider of crucial importance the capability to detect the presence of core and supportive clinical features to generate appropriate categories of probable or possible DLB. Nevertheless, it has been clearly shown that the possibility of a wrong clinical diagnosis in clinical practice remains a concern due to difficulties to detect such clinical features especially in the early phase at onset. With this respect, the diagnostic evaluation of a potential DLB patient requires careful interview which is not limited to the patient, to pickup supportive symptoms such as constipation and hyposmia, but it should include also the relatives and the partner to ascertain insidious core symptoms such as RBD and cognitive fluctuations. Moreover, careful clinical examination is mandatory to disclose $\mathrm{OH}$ and parkinsonism. According to diagnostic criteria for DLB since their first formulation dementia is by definition an essential requirement for DLB diagnosis, while criteria for prodromal DLB, albeit in preparation, are not yet available. This means that the diagnosis of DLB in pre-dementia stages can currently be hypothesized, but not made with the help of a formal support. Furthermore, also clinical criteria for detection of patients previously characterized as $\mathrm{LB}$ variant of $\mathrm{AD}$ remain to be formulated.

Second, DLB Consortium in the fourth Consensus report clearly states that clinical diagnosis of DLB in clinical routine is suboptimal and gives more weight to molecular imaging biomarkers to offer a more objective and consistently reliable information. Indeed, current knowledge toward the pathological substrates of DLB clearly shows that available molecular imaging targets including the DaT uptake in the basal ganglia, the NeT uptake in sympathetic myocardial tissue and the cerebral glucose metabolism, can be considered reliable and high-value indices for the objective evaluation and diagnosis of DLB directly in vivo. Although the definition of DLB was not divorced from clinical symptoms, the attempt to define DLB by biomarkers indicative of pathophysiological changes together with clinical core features represents a profound shift in thinking.

Third, along with DaT, mIBG and FDG imaging techniques, molecular imaging using brain PET with more specific radiotracers could open a new scenario for an accurate 
evaluation of biomarkers involved in DLB. Indeed, although direct biomarker evidence of LB-related pathology is not yet available, there is an increasing evidence that amyloid and tau PET may play a role in the diagnosis of DLB. Mirroring the findings of pathologic studies which reveal the common concomitance of AD plaque changes, PET with amyloid tracers is often positive in subjects with DLB. On the other hand, in light of pathologic studies showing only occasional presence of neurofibrillary tangles in the neocortex, a negative neocortical uptake should contribute to favor a diagnosis of DLB over AD. Conversely, although a positive neocortical uptake is not necessarily more in favor of an AD than a DLB diagnosis, the cortical tau aggregates was shown to be associated with severity of cognitive impairment and to be present even in those DLB subjects without elevated amyloid levels. Thus, according with molecular imaging-pathological model, the use of amyloid or tau-imaging procedures could represent an additional attempt to define DLB by biomarkers indicative of neuropathologic changes.

Fourth, although no approved or shared recommendation on which indicative biomarker is to be used with respect to patient's clinical presentation, an indicative biomarker unrelated to the corresponding clinical feature should preferentially be used to overcome circularity.

Finally, it has been shown that in vivo quantitative assessment of time-activity concentration of functional parameters allows to quantify a number of processes (e.g., receptor binding, receptor occupancy, rate of glucose utilization, and accumulation of pathologic proteins) and to link the resulting estimates to clinical parameters (e.g., disease severity, disease evolution, response to treatment, and survival). Importantly, such resulting estimates have been proven to increase diagnostic accuracy of neurodegenerative disease including DLB and to play a role for personalized medicine to patient management. Along with molecular imaging, quantitative analysis tools are also important for MRI. Indeed, although diagnostic utility of MRI is often thought to lie in its exclusionary role of secondary causes of dementia, it is to highlight that quantitative estimate of severity of MTL atrophy and of cerebrovascular disease may serve to differentiate DLB from AD and vascular dementia, respectively.

\section{Compliance with ethical standards}

Conflict of interest The author declares no conflicts of interest; this paper does not contain results of studies performed by the author.

\section{References}

1. Jack CR Jr, Bennett DA, Blennow K et al (2018) NIA-AA research framework: toward a biological definition of Alzheimer's disease. Alzheimer Dement 14(4):535-562
2. Okazaki H, Lipkin LE, Aronson SM (1961) Diffuse intracytoplasmic ganglionic inclusions (Lewy type) associated with progressive dementia and quadriparesis in flexion. J Neuropathol Exp Neurol 20:237-244

3. McKhann G, Drachman D, Folstein M, Katzman R, Price D, Stadlan EM (1984) Clinical diagnosis of Alzheimer's disease: report of the NINCDS-ADRDA Work Group under the auspices of Department of Health and Human Services Task Force on Alzheimer's Disease. Neurology 34:939-944

4. McKeith IG, Galasko D, Kosaka K et al (1996) Consensus guidelines for the clinical and pathologic diagnosis of dementia with Lewy bodies (DLB): report of the consortium on DLB international workshop. Neurology 47(5):1113-1124

5. Vann Jones SA, O'Brien JT (2014) The prevalence and incidence of dementia with Lewy bodies: a systematic review of population and clinical studies. Psychol Med 44:673-683

6. Sakamoto F, Shiraishi S, Tsuda N, Hashimoto M, Tomiguchi S, Ikeda M, Yamashita Y (2017) Diagnosis of dementia with Lewy bodies: can ${ }^{123}$ I-IMP and ${ }^{123}$ I-MIBG scintigraphy yield new core features? Br J Radiol 90(1070):1-8

7. Hogan DB, Fiest KM, Roberts JI, Maxwell CJ, Dykeman J, Pringsheim T, Steeves T, Smith EE, Pearson D, Jetté N (2016) The prevalence and incidence of dementia with Lewy bodies: a systematic review. Can J Neurol Sci 43(S1):S83-S95

8. Gauthier S, Zhang H, Ng KP, Pascoal TA, Rosa-Neto P (2018) Impact of the biological definition of Alzheimer's disease using amyloid, tau and neurodegeneration (ATN): what about the role of vascular changes, inflammation, Lewy body pathology? Transl Neurodegen $7: 1-12$

9. McKeith IG, Dickson DW, Lowe J et al (2005) Diagnosis and management of dementia with Lewy bodies: third report of the DLB Consortium. Neurology 65(12):1863-1872

10. McKeith IG, Boeve BF, Dickson DW et al (2017) Diagnosis and management of dementia with Lewy bodies: Fourth consensus report of the DLB Consortium 89(1):88-100

11. Mukaetova-Ladinska EB, McKeith IG (2006) Pathophysiology of synuclein aggregation in Lewy body disease. Mech Ageing Dev 127:188-202

12. Hansen LA (1997) The Lewy body variant of Alzheimer disease. J Neural Transm Suppl 51:83-93

13. Ballard C, Ziabreva I, Perry R, Larsen JP, O’Brien J, McKeith I, Perry E, Aarsland D (2006) Differences in neuropathologic characteristics across the Lewy body dementia spectrum. Neurology 67:1931-1934

14. Grothe MJ, Schuster C, Bauer F, Heinsen H, Prudlo J, Teipel SJ (2014) Atrophy of the cholinergic basal forebrain in dementia with Lewy bodies and Alzheimer's disease dementia. J Neurol 261:1939-1948

15. Jellinger KA, Attems J (2008) Prevalence and impact of vascular and Alzheimer pathologies in Lewy body disease. Acta Neuropathol 115:427-436

16. Braak H, Braak E (1991) Neuropathological stageing of Alzheimer-related changes. Acta Neuropathol 82(4):239-259

17. Tiraboschi P, Attems J, Thomas A, Brown A, Jaros E, Lett DJ, Ossola M, Perry RH, Ramsay L, Walker L, McKeith IG (2015) Clinicians' ability to diagnose dementia with Lewy bodies is not affected by beta-amyloid load. Neurology 84:496-499

18. Toledo JB, Gopal P, Raible K et al (2016) Pathological alphasynuclein distribution in subjects with coincident Alzheimer's and Lewy body pathology. Acta Neuropathol 131:393-409

19. Gomperts SN, Locascio JJ, Makaretz SJ et al (2016) Tau positron emission tomographic imaging in the Lewy body diseases. Jama Neurol 73:1334-1341

20. Goldman JG, Williams-Gray C, Barker RA, Duda JE, Galvin JE (2014) The spectrum of cognitive impairment in Lewy body diseases. Mov Disord 29(5):608-621 
21. Hohl U, Tiraboschi P, Hansen LA, Thal LJ, Corey-Bloom J (2000) Diagnostic accuracy of dementia with Lewy bodies. Arch Neurol 57(3):347-351

22. Galvin JE, Duda JE, Kaufer DI, Lippa CF, Taylor A, Zarit SH (2010) Lewy body dementia: the caregiver experience of clinical care. Parkinsonism Relat Disord 16(6):388-392

23. Högl B, Stefani A, Videnovic A (2018) Idiopathic REM sleep behaviour disorder and neurodegeneration-an update. Nat Rev Neurol 14:40-55

24. Barone DA, Henchcliffe C (2018) Rapid eye movement sleep behavior disorder and the link to alpha-synucleinopathies. Clin Neurophysiol 129(8):1551-1564

25. Boeve BF, Molano JR, Ferman TJ et al (2011) Validation of the Mayo Sleep Questionnaire to screen for REM sleep behavior disorder in an aging and dementia cohort. Sleep Med 12(5):445-453

26. Ballard Grace J, McKeith I, Holmes C (1998) Neuroleptic sensitivity in dementia with Lewy bodies and Alzheimer's disease. Lancet 351:1032-1033

27. Doty RL (1989) Influence of age and age-related diseases on olfactory function. Ann N Y Acad Sci 561:76-86

28. Briner HR, Simmen D (1999) Smell diskettes as screening test of olfaction. Rhinology 37(4):145-148

29. Walker MP, Ayre GA, Cummings JL, Wesnes K, McKeith IG, O'Brien JT, Ballard CG (2000) The clinician assessment of fluctuation and the one day fluctuation assessment scale. Two methods to assess fluctuating confusion in dementia. Br J Psychiatry 177:252-256

30. Postuma RB, Berg D, Stern M et al (2015) MDS clinical diagnostic criteria for Parkinson's disease. Mov Disord 30:1591-1601

31. Donaghy PC, McKeith IG (2014) The clinical characteristics of dementia with Lewy bodies and a consideration of prodromal diagnosis. Alzheimers Res Ther 6(4):46-58

32. Rizzo G, Arcuti S, Copetti M, Alessandria M, Savica R, Fontana A, Liguori R, Logroscino G (2018) Accuracy of clinical diagnosis of dementia with Lewy bodies: a systematic review and meta-analysis. J Neurol Neurosurg Psychiatry 89(4):358-366

33. Stinton C, McKeith I, Taylor JP et al (2015) Pharmacological management of Lewy body dementia: a systematic review and meta-analysis. Am J Psychiatry 172(8):731-774

34. Walker Z, Costa DC, Ince P, McKeith IG, Katona CL (1999) Invivo demonstration of dopaminergic degeneration in dementia with Lewy bodies. Lancet 354(9179):646-647

35. Walker Z, Costa DC, Walker RW et al (2002) Differentiation of dementia with Lewy bodies from Alzheimer's disease using a dopaminergic presynaptic ligand. J Neurol Neurosurg Psychiatry 73(2):134-340

36. Tabet N, Walker Z, Mantle D, Costa D, Orrell M (2003) In vivo dopamine pre-synaptic receptors and antioxidant activities in patients with Alzheimer's disease, dementia with Lewy bodies and in controls. A preliminary report. Dement Geriatr Cogn Disord 16(1):46-51

37. Walker RW, Walker Z (2009) Dopamine transporter single photon emission computerized tomography in the diagnosis of dementia with Lewy bodies. Mov Disord 24(Suppl 2):S754-S759

38. McKeith I, O'Brien J, Walker Z et al (2007) Sensitivity and specificity of dopamine transporter imaging with 123I-FP-CIT SPECT in dementia with Lewy bodies: a phase III, multicentre study. Lancet Neurol 6(4):305-313

39. O'Brien JT, McKeith IG, Walker Z et al (2009) Diagnostic accuracy of 123I-FP-CIT SPECT in possible dementia with Lewy bodies. Br J Psychiatry 194(1):34-39

40. Walker Z, Jaros E, Walker RW et al (2007) Dementia with Lewy bodies: a comparison of clinical diagnosis, FP-CIT single photon emission computed tomography imaging and autopsy. J Neurol Neurosurg Psychiatry 78(11):1176-1181
41. Walker Z, Costa DC, Walker RW et al (2004) Striatal dopamine transporter in dementia with Lewy bodies and Parkinson disease: a comparison. Neurology 62(9):1568-1572

42. Costa DC, Walker Z, Walker RW, Fontes FR (2003) Dementia with Lewy bodies versus Alzheimer's disease: role of dopamine transporter imaging. Mov Disord 18(Suppl 7):S34-S38

43. Walker Z, Cummings JL (2012) [123I] N- $\omega$-fluoropropyl-2 $\beta$ carbomethoxy-3 $\beta$-(4-iodophenyl) nortropane single-photon emission computed tomography brain imaging in the diagnosis of dementia with Lewy bodies. Alzheimers Dement 8(1):74-83

44. Grosset DG, Tatsch K, Oertel WH et al (2014) Safety analysis of 10 clinical trials and for 13 years after first approval of ioflupane 123I injection (DaTscan). J Nucl Med 55(8):1281-1287

45. Walker Z, Moreno E, Thomas A et al (2015) Clinical usefulness of dopamine transporter SPECT imaging with 123I-FP-CIT in patients with possible dementia with Lewy bodies: randomised study. Br J Psychiatry 206(2):145-152

46. Walker Z, Possin KL, Boeve BF (2015) Lewy body dementias. Lancet 386(10004):1683-1697

47. Walker Z, Moreno E, Thomas A et al (2016) Evolution of clinical features in possible DLB depending on FP-CIT SPECT result. Neurology 87(10):1045-1051

48. Thomas AJ, Attems J, Colloby SJ et al (2017) Autopsy validation of 123I-FP-CIT dopaminergic neuroimaging for the diagnosis of DLB. Neurology 88(3):276-283

49. Papathanasiou ND, Boutsiadis A, Dickson J, Bomanji JB (2012) Diagnostic accuracy of ${ }^{123}$ I-FP-CIT (DaTSCAN) in dementia with Lewy bodies: a meta-analysis of published studies. Parkinsonism Relat Disord 18(3):225-229

50. McCleery J, Morgan S, Bradley KM, Noel-Storr AH, Ansorge O, Hyde C (2015) Dopamine transporter imaging for the diagnosis of dementia with Lewy bodies. Cochrane Database Syst Rev 1:CD010633

51. Brigo F, Turri G, Tinazzi M (2015) 123I-FP-CIT SPECT in the differential diagnosis between dementia with Lewy bodies and other. J Neurol Sci 359(1-2):161-171

52. O'Brien JT, Colloby S, Fenwick J et al (2004) Dopamine transporter loss visualized with FP-CIT SPECT in the differential diagnosis of dementia with Lewy bodies. Arch Neurol 61(6):919-925

53. Morgan S, Kemp P, Booij J et al (2012) Differentiation of frontotemporal dementia from dementia with Lewy bodies using FPCIT SPECT. J Neurol Neurosurg Psychiatry 83(11):1063-1070

54. Eshuis SA, Jager PL, Maguire RP, Jonkman S, Dierckx RA, Leenders KL (2009) Direct comparison of FP-CIT SPECT and F-DOPA PET in patients with Parkinson's disease and healthy controls. Eur J Nucl Med Mol Imaging 36(3):454-462

55. Yoshita M, Taki J, Yokoyama K et al (2006) Value of 123I-MIBG radioactivity in the differential diagnosis of DLB from AD. Neurology 66(12): 1850-1854

56. Treglia G, Cason E, Cortelli P et al (2014) Iodine-123 metaiodobenzylguanidine scintigraphy and iodine-123 ioflupane single photon emission computed tomography in Lewy body diseases: complementary or alternative techniques? J Neuroimaging 24(2):149-154

57. Braune S (2001) The role of cardiac metaiodobenzylguanidine uptake in the differential diagnosis of parkinsonian syndromes. Clin Auton Res 11(6):351-355

58. Treglia G, Cason E (2012) Diagnostic performance of myocardial innervation imaging using MIBG scintigraphy in differential diagnosis between dementia with Lewy bodies and other dementias: a systematic review and a meta-analysis. J Neuroimaging 22(2):111-117

59. King AE, Mintz J, Royall DR (2011) Meta-analysis of 123I-MIBG cardiac scintigraphy for the diagnosis of Lewy bodyrelated disorders. Mov Disord 26(7):1218-1224 
60. Braune S, Reinhardt M, Schnitzer R, Riedel A, Lücking $\mathrm{CH}$ (1999) Cardiac uptake of [123I]MIBG separates Parkinson's disease from multiple system atrophy. Neurology 53(5):1020

61. Nuvoli S, Palumbo B, Malaspina S, Madeddu S, Spanu A (2018) ${ }^{123}$ I-ioflupane SPET and 123I-MIBG in the diagnosis of Parkinson's disease and parkinsonian disorders and in the differential diagnosis between Alzheimer's and Lewy's bodies dementias. Hell J Nucl Med 21(1):60-68

62. Surendranathan A, O'Brien JT (2018) Clinical imaging in dementia with Lewy bodies. Evid Based Mental Health 21(2):61-65

63. Jellinger KA (2018) Dementia with Lewy bodies and Parkinson's disease-dementia: current concepts and controversies. J Neural Transm 125:615-650

64. Tiraboschi P, Corso A, Guerra UP et al (2016) (123) I-2 $\beta$ carbomethoxy-3 $\beta$-(4-iodophenyl)-N-(3-fluoropropyl) nortropane single photon emission computed tomography and (123) I-metaiodobenzylguanidine myocardial scintigraphy in differentiating dementia with lewy bodies from other dementias: a comparative study. Ann Neurol 80(3):368-378

65. Thobois S, Prange S, Scheiber C, Broussolle E (2018) What a neurologist should know about PET and SPECT functional imaging for parkinsonism: a practical perspective. Parkinson Relat Disord S1353-8020(18):30375-4

66. Orimo S, Amino T, Itoh Y et al (2005) Cardiac sympathetic denervation precedes neuronal loss in the sympathetic ganglia in Lewy body disease. Acta Neuropathol 109(6):583-588

67. Sakakibara R, Tateno F, Kishi M, Tsuyusaki Y, Terada H, Inaoka T (2014) MIBG myocardial scintigraphy in pre-motor Parkinson's disease: a review. Parkinsonism Relat Disord 20(3):267-273

68. Gabilondo I, Llorens V, Rodriguez T et al (2018) Myocardial MIBG scintigraphy in genetic Parkinson's disease as a model for Lewy body disorders. EJNMMI. https://doi.org/10.1007/s0025 9-018-4183-0

69. Komatsu J, Samuraki M, Nakajima K et al (2018) ${ }^{123}$ I-MIBG myocardial scintigraphy for the diagnosis of DLB: a multicentre 3-year follow-up study. J Neurol Neurosurg Psychiatry 89(11):1167-1173

70. Suzuki M, Kurita A, Hashimoto M et al (2006) Impaired myocardial 123I-metaiodobenzylguanidine uptake in Lewy body disease: comparison between dementia with Lewy bodies and Parkinson's disease. J Neurol Sci 240:15-19

71. Yoshita M, Arai H, Arai H et al (2015) Diagnostic accuracy of 123I-meta-iodoben-zylguanidine myocardial scintigraphy in dementia with Lewy bodies: a multicenter study. PLoS One 10(3): $1-10$

72. Timóteo da Rocha E, Alves WEFA, Verschure DO, Verberne HJ (2017) The use of cardiac ${ }^{123}$ I-mIBG scintigraphy in clinical practice: the necessity to standardize! Int J Cardiovasc Sci 30(6):533-541

73. Odagiri H, Baba T, Nishio Y et al (2016) On the utility of MIBG SPECT/CT in evaluating cardiac sympathetic dysfunction in Lewy body diseases. PloS One 7:1-10

74. Saeed U, Compagnone J, Aviv RI et al (2017) Imaging biomarkers in Parkinson's disease and Parkinsonian syndromes: current and emerging concepts. Transl Neurodegen 6:8-33

75. Liu S, Wang Xiao-Dan, Wang Ying et al (2017) Clinical and neuroimaging characteristics of Chinese dementia with Lewy bodies. PLoS One 12(3):e0171802

76. Higuchi M, Tashiro M, Arai H, Okamura N, Hara S, Higuchi S, Itoh M, Shin RW, Trojanowski JQ, Sasaki H (2000) Glucose hypometabolism and neuropathological correlates in brains of dementia with Lewy bodies. Exp Neurol 162(2):247-256

77. Minoshima S, Foster NL, Sima AA, Frey KA, Albin RL, Kuhl DE (2001) Alzheimer's disease versus dementia with Lewy bodies: cerebral metabolic distinction with autopsy confirmation. Ann Neurol 50(3):358-365

78. Lim SM, Katsifis A, Villemagne VL et al (2009) The 18F-FDG PET cingulate island sign and comparison to 123I-beta-CIT SPECT for diagnosis of dementia with Lewy bodies. J Nucl Med 50(10):1638-1645

79. Imamura T, Ishii K, Sasaki M et al (1997) Regional cerebral glucose metabolism in dementia with Lewy bodies and Alzheimer's disease: a comparative study using positron emission tomography. Neurosci Lett 235(1-2):49-52

80. Yousaf T, Dervenoulas G, Valkimadi PE, Politis M (2018) Neuroimaging in Lewy Body dementia. J Neurol. https://doi org/10.1007/s00415-018-8892-x

81. Graff-Radford J, Murray ME, Lowe VJ et al (2014) Dementia with Lewy bodies: basis of cingulate island sign. Neurology 83(9):801-809

82. Fiorenzato E, Biundo R, Cecchin D, Frigo AC, Kim J, Weis L, Strafella AP, Antonini A (2018) Brain amyloid contribution to cognitive dysfunction in early-stage Parkinson's Disease: the PPMI dataset. J Alzheimers Dis 66(1):229-237

83. Whitwell JL, Whitwell JL, Graff-Radford J, Singh TD et al (2017) ${ }^{18}$ F-FDG PET in posterior cortical atrophy and dementia with lewy bodies. J Nucl Med 58(4):632-638

84. Saeed U, Compagnone J, Aviv RI, Strafella AP, Black SE, Lang AE, Masellis M (2017) Imaging biomarkers in Parkinson's disease and Parkinsonian syndromes: current and emerging concepts. Transl Neurodegener 6:8

85. Frey KA, Petrou M (2015) Imaging amyloidopathy in parkinson disease and Parkinsonian dementia syndromes. Clin Transl Imaging 3(1):57-64

86. Gomperts SN (2014) Imaging the role of amyloid in PD dementia and dementia with Lewy bodies. Curr Neurol Neurosci Rep 14(8):472

87. Maetzler W, Liepelt I, Reimold M et al (2009) Cortical PIB binding in Lewy body disease is associated with Alzheimer-like characteristics. Neurobiol Dis 34(1):107-112

88. Gomperts SN, Rentz DM, Moran E et al (2008) Imaging amyloid deposition in Lewy body diseases. Neurology 71(12):903-910

89. Kantarci K, Lowe VJ, Boeve BF et al (2012) Multimodality imaging characteristics of dementia with Lewy bodies. Neurobiol Aging 33(9):2091-2105

90. Lee SH, Cho H, Choi JY, Lee JH, Ryu YH, Lee MS, Lyoo CH (2018) Distinct patterns of amyloid-dependent tau accumulation in Lewy body diseases. Mov Disord 33(2):262-272

91. Bohnen NI, Müller MLTM, Frey KA (2017) Molecular imaging and updated diagnostic criteria in Lewy body dementias. Curr Neurol Neurosci Rep 17(10):73

92. Kantarci K, Lowe VJ, Boeve BF et al (2017) AV-1451 tau and $\beta$-amyloid positron emission tomography imaging in dementia with Lewy bodies. Ann Neurol 81(1):58-67

93. Hansen AK, Damholdt MF, Fedorova TD et al (2017) In Vivo cortical tau in Parkinson's disease using 18F-AV-1451 positron emission tomography. Mov Disord 32(6):922-927

94. Bauckneht M, Arnaldi S, Nobili F, Aarsland A, Morbelli S (2018) New tracers and new perspectives for molecular imaging in Lewy body disease. Curr Med Chem 25:3105-3130

95. Lockhart A, Davis B, Matthews JC et al (2003) The peripheral benzodiazepine receptor ligand PK11195 binds with high affinity to the acute phase reactant alpha1-acid glycoprotein: implication for the use of the ligand as a CNS inflammatory marker. Nucl Med Biol 30(2):199-206

96. Surendranathan A, Su L, Passamonti L et al (2017) [11C] PK11195 PET imaging reveals neuroinflammation in dementia with Lewy Body is negatively associated with disease severity: NIMROD study. Alzheimer Dement J 13(7):p64-p65 
97. Surendranathan A, Rowe JB, O’Brine JT (2015) Neuroinflammation in Lewy Body dementia. Parkinsonism Relat Disord 21(12):1398-1406

98. International Classification of Sleep Disorders (2005) Diagnostic and coding manual, 2nd edn. American Academy of Sleep Medicine, Westchester

99. Cromarty RA, Elder GJ, Graziadio S et al (2016) Neurophysiological biomarkers for Lewy body dementias. Clin Neurophysiol 127(1):349-359

100. Bonanni L, Perfetti B, Bifolchetti S et al (2015) Quantitative electroencephalogram utility in predicting conversion in mild cognitive impairment to dementia with Lewy bodies. Neurobiol Aging 36(1):434-445

101. Filippi M, Agosta F, Barkhof F et al (2012) EFNS task force: the use of neuroimaging in the diagnosis of dementia. Eur J Neurol 19(12):e131-e140

102. Oppedal K, Ferreira D, Cavallin L et al (2018) A signature pattern of cortical atrophy in dementia with Lewy bodies: a study on 333 patients from the European DLB consortium. Alzheimers Dement 15:1-10

103. Burton EJ, Barber R, Mukaetova-Ladinska EB, Robson J, Perry RH, Jaros E, Kalaria RN, O'Brien JT (2009) Medial temporal lobe atrophy on MRI differentiates Alzheimer's disease from dementia with Lewy bodies and vascular cognitive impairment: a prospective study with pathological verification of diagnosis. Brain 132(Pt 1):195-203

104. Harper L, Fumagalli GG, Barkhof F et al (2016) MRI visual rating scales in the diagnosis of dementia: evaluation in 184 postmortem confirmed cases. Brain 139(4):1211-1225

105. Burton EJ, Mukaetova-Ladinska EB, Perry RH, Jaros E, Barber R, O'Brien JT (2012) Neuropathological correlates of volumetric MRI in autopsy-confirmed Lewy body dementia. Neurobiol Aging 33(7):1228-1236

106. Nedelska Z, Ferman TJ, Boeve BF et al (2015) Pattern of brain atrophy rates in autopsy-confirmed dementia with Lewy bodies. Neurobiol Aging 36(1):452-461

107. Abdelnour C, van Steenoven I, Londos E et al (2016) Alzheimer's disease cerebrospinal fluid biomarkers predict cognitive decline in lewy body dementia. Mov Disord 31(8):1203-1208

108. Mollenhauer B, Cullen V, Kahn I et al (2008) Direct quantification of CSF alpha-synuclein by ELISA and first cross-sectional study in patients with neurodegeneration. Exp Neurol 213(2):315-325

109. Oskar Hansson O, Hall S, Öhrfelt A et al (2014) Levels of cerebrospinal fluid $\alpha$-synuclein oligomers are increased in Parkinson's disease with dementia and dementia with Lewy bodies compared to Alzheimer's disease. Alzheimer's Res Ther 6:25

110. Donadio V, Incensi A, Rizzo G et al (2017) A new potential biomarker for dementia with Lewy bodies: skin nerve $\alpha$-synuclein deposits. Neurology 89(4):318-326

111. Guerreiro R, Ross OA, Kun-Rodrigues C et al (2018) Investigating the genetic architecture of dementia with Lewy bodies: a twostage genome-wide association study. Lancet Neurol 17(1):64-74

112. Slaets S, Van Acker F, Versijpt J et al (2015) Diagnostic value of MIBG cardiac scintigraphy for differential dementia diagnosis. Int J Geriatr Psychiatry 30:864-869

113. Jacobson AF, Travin MI (2015) Impact of medications on mIBG uptake, with specific attention to the heart: comprehensive review of the literature. J Nucl Cardiol 22(5):980-993

114. Del Sole A, Perini G, Lecchi M et al (2015) Correlation between 123I-FP-CIT brain SPECT and parkinsonism in dementia with Lewy bodies: caveat for clinical use. Clin Nucl Med 40:32-35

115. Kobayashi S, Makino K, Hatakeyama S et al (2017) The usefulness of combined brain perfusion single-photon emission computed tomography, Dopamine-transporter single-photon emission computed tomography, and ${ }^{123}$ I-metaiodobenzylguanidine myocardial scintigraphy for the diagnosis of dementia with Lewy bodies. J Neurol 14:247-255

116. Nobili F, Arbizu J, Bouwman F et al (2018) European Association of Nuclear Medicine and European Academy of Neurology recommendations for the use of brain ${ }^{18} \mathrm{~F}$-fluorodeoxyglucose positron emission tomography in neurodegenerative cognitive impairment and dementia: Delphi consensus. Eur J Neurol 25(10):1201-1217

Publisher's Note Springer Nature remains neutral with regard to jurisdictional claims in published maps and institutional affiliations. 